Chronic Obstructive Pulmonary Diseases:

Journal of the COPD Foundation

COPD

FOUNDATION

\title{
Journal Club \\ New Treatment Options for COPD: How Do We Decide Phenotypes, Endotypes or Treatable Traits?
}

\author{
Ron Balkissoon, MD, MSc, DIH, FRCPC ${ }^{1}$
}

\begin{abstract}
Abbreviations: Global initiative for chronic Obstructive Lung Disease, GOLD; chronic obstructive pulmonary disease, COPD; long-acting muscarinc antagonists, LAMAs; long-acting beta2-agonists, LABAs; inhaled corticosteroids, ICSs; interleukin-5, IL-5; beclometasone dipropionate, BDP; formoterol fumarate, FF; COPD Assessment test, CAT; confidence interval, CI; forced expiratory volume in 1 second, FEV1; budesonide, BUD; formoterol, FOR

Citation: Balkissoon R. Journal club. New treatments options for COPD: How do we decide phenotypes, endotypes or treatable traits? Chronic Obstr Pulm Dis. 2018;5(1):72-80. doi: https://doi.org/10.15326/jcopdf.5.1.2018.0128
\end{abstract}

1 Denver, Colorado

\section{Address correspondence to:}

Ron Balkissoon, MD, MSc, DIH, FRCPC

balkissoonr@njhealth.org

\section{Introduction}

In 2017, the Global initiative for chronic Obstructive Lung Disease (GOLD) guidelines introduced significant changes to the classification and treatment recommendations for chronic obstructive pulmonary disease (COPD) patients. ${ }^{1}$ Lung function was no longer incorporated in the "A, B, C, D" classification scheme and maximizing bronchodilation first and foremost was given priority. Other than Group A patients, longacting muscarinc antagonists (LAMAs) and/or longacting beta2-agonists (LABAs) were recommended as first line across all groups before considering the use of inhaled corticosteroids (ICS)/LABA combination therapy (ICS/LABA) or triple therapy (ICS/LABA/ LAMA). The exceptions to this were patients who were deemed to be asthma/COPD overlap patients and/ or demonstrated evidence of persistent sputum or peripheral blood eosinophilia regardless of any prior history of asthma. ${ }^{2-6}$ For these patients, ICS/LABA combinations were proposed as an appropriate first line therapy, particularly in light of the boxed warning indicating that asthma patients should be treated first line with controller medications such as ICSs. This change in the treatment paradigm was largely driven by the concerns that inhaled ICSs were associated with an increased risk of pneumonia ${ }^{7-11}$ and could exacerbate other comorbidities such as cataracts, osteopenia, and diabetes. Additionally, there were several studies suggesting that LAMA and LABA/LAMA combination therapies were able to reduce exacerbations in patients who had experienced at least one exacerbation in the prior 12 months. ${ }^{12-17}$ Globally, in the past 12 to 24 months we have seen the approval of several dual LABA/LAMA bronchodilators and 2 single inhaler triple combinations of ICS/LABA/LAMA for treatment of COPD. We have recently also had the U.S. approval of a nebulized twice daily LAMA, glycopyrrolate, and there is a nebulized once daily LAMA, revefenacin, that has been submitted for a new drug application to the U.S. Food and Drug Administration as of November 2017. These new nebulized LAMAs will allow appropriate COPD patients to be able to have complete nebulized triple therapy. Another study this year examined the potential role for the anti-interleukin-5 (IL-5) agent, mepolizumab, as a treatment option for COPD patients. Mepolizumab has been approved for treatment of severe asthma that is refractory to standard controller therapies such as ICSs or requires high-dose ICSs and/or systemic oral corticosteroids to remain controlled. Studies have demonstrated that its greatest efficacy was found in enriched cohorts with persistent eosinophilia despite the use of inhaled and/or systemic corticosteroids. A previous study that examined benralizumab in COPD had not shown a significant reduction in exacerbations but there did seem to be an increased response associated with higher sputum eosinophil counts. ${ }^{18}$ 
In both asthma and COPD there is great interest in identifying phenotypes and endotypes that allow for precise, targeted therapies based on clinical characteristics and biomarkers such as eosinophils. In COPD, where multiple comorbidities can contribute to COPD exacerbations, the prospect of trying to define the various permutations and combinations of these clinical characteristics to define phenotypes/endotypes can seem daunting. The prospect of then trying to take such a matrix and overlay a treatment paradigm of the various combination medications that have become available in the last 24 months is even more overwhelming. In this context, the concept of "treatable traits" has been proposed. An example of this is the peripheral blood eosinophil count and its potential role in identifying patients who may be candidates for ICSs and/or anti-IL-5 agents over and above maximum bronchodilation. Another clinical feature that seems to be a potentially important "treatable trait" is frequent moderate and severe exacerbations. Peter Calverley reviewed data from a trial that compared ICS/LABA to LAMA/LAMA and suggested that patients with eosinophil counts greater than $300 / \mu \mathrm{L}$ and 2 or more moderate or 1 severe exacerbation(s) in the prior 12 months seemed to be better responders to ICS/LABA than LABA/LAMA. ${ }^{19}$

Hence, it is a very exciting and dynamic time in the field of COPD research and clinical care and the options for treating our patients seem to be growing exponentially. With so many options, trying to make sense of how to make decisions regarding treatment is becoming more challenging. In this edition of the Journal Club, I review the recent papers on the clinical efficacy of single triple inhaler therapies and anti-IL- 5 agents in the treatment of COPD patients. As you will note, further research is certainly required to define the exact role for these agents in light of the continually evolving understanding of the COPD patient population.

Note: Abstracts are presented in their original, published format and have not been edited to match JCOPDF style.

\section{Abstract 1 Single Inhaler Triple Therapy Versus Inhaled Corticosteroid Plus Long- Acting B2-Agonist Therapy for Chronic Obstructive Pulmonary Disease (TRILOGY): A Double- Blind, Parallel Group, Randomised Controlled Trial}

Singh D, Papi A, Corradi M, et al. Lancet. 2016;388(10 048):963-973.

doi: https://doi.org/10.1016/S0140-6736(16)31354-X

\section{BACKGROUND:}

Few data are available for the efficacy of "triple therapy" with two long-acting bronchodilators and an inhaled corticosteroid in chronic obstructive pulmonary disease (COPD). We designed this study to assess efficacy of single-inhaler combination of an extra fine formulation of beclometasone dipropionate, formoterol fumarate, and glycopyrronium bromide (BDP/FF/GB) in COPD compared with beclometasone dipropionate and formoterol fumarate (BDP/FF) treatment.

\section{METHODS:}

TRILOGY was a randomised, parallel group, doubleblind, active-controlled study done in 159 sites across 14 countries. The sites were a mixture of primary, secondary, and tertiary care providers, and specialist investigation units. Eligible patients with COPD had post-bronchodilator forced expiratory volume in 1 $s\left(F E V_{1}\right)$ of lower than $50 \%$, one or more moderateto-severe COPD exacerbation in the previous 12 months, COPD Assessment Test total score of 10 or more, and a Baseline Dyspnea Index focal score of 10 or less. Patients who met the inclusion and exclusion criteria at screening entered a 2-week open-label run-in period where they received beclometasone dipropionate $(100 \mu \mathrm{g})$ and formoterol fumarate $(6 \mu \mathrm{g})$ in two actuations twice daily. Patients were then randomly assigned (1:1) with an interactive response technology system to either continue BDP (100 $\mu \mathrm{g})$

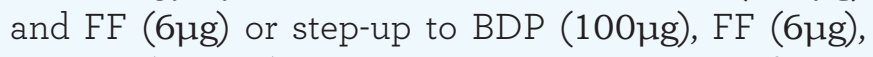
and $\mathrm{GB}(12.5 \mu \mathrm{g})$ in two actuations twice daily for 52 weeks via pressurised metered-dose inhaler. The three co-primary endpoints were pre-dose $\mathrm{FEV}_{1}$, 2-h post- 
dose $\mathrm{FEV}_{1}$, and Transition Dyspnea Index (TDI) focal score, all measured at week 26 in the intention-to-treat population (all patients who were randomly assigned and received at least one dose of study drug and had at least one post-baseline efficacy assessment). Safety outcomes were measured in the safety population (all patients who were randomly assigned and received at least one dose of study drug). Secondary endpoints included moderate-to severe COPD exacerbation rate over 52 weeks.

\section{FINDINGS:}

Between March 21, 2014, and Jan 14, 2016, 1368 patients received either $\mathrm{BDP} / \mathrm{FF} / \mathrm{GB}(\mathrm{n}=687)$ or $\mathrm{BDP} /$ FF ( $\mathrm{n}=681)$. At week 26, BDP/FF/GB improved predose FEV 1 by $0.081 \mathrm{~L}(95 \%$ CI 0.052-0.109; $p<0.001)$ and 2-h post-dose $\mathrm{FEV}_{1}$ by $0.117 \mathrm{~L}$ (0.086-0.147; $p<0.001)$ compared with BDP/FF. Mean TDI focal scores at week 26 were 1.71 for BDP/FF/GB and 1.50 for BDP/FF, with a difference of 0.21 (95\% CI -0.08 to $0.51 ; p=0.160)$. Adjusted annual moderate-to-severe exacerbation frequencies were 0.41 for BDP/FF/GB and 0.53 for BDP/FF (rate ratio 0.77 [95\% CI 0.650.92 ]; $p=0.005$ ), corresponding to a $23 \%$ reduction in exacerbations with $\mathrm{BDP} / \mathrm{FF} / \mathrm{GB}$ compared with BDP/FF. Adverse events were reported by 368 (54\%) patients with BDP/FF/GB and 379 (56\%) with BDP/ FF. One serious treatment-related adverse event occurred (atrial fibrillation) in a patient in the BDP/ FF/GB group.

\section{INTERPRETATION:}

We provide evidence for the clinical benefits of stepping up patients with COPD from an inhaled corticosteroid/long-acting $\beta 2$-agonist combination treatment to triple therapy using a single inhaler.

FUNDING: Chiesi Farmaceutici SpA.

\section{Comments}

The TRILOGY study focused on lung function and symptoms as the primary endpoints. Interestingly, the lung function results reported are at week 26 where there was an $81 \mathrm{~mL}$ difference between the 2 treatment arms versus week 52 where the difference was only $63 \mathrm{~mL}$. While these differences are statistically significant they are less than the typically recognized minimal clinical important difference of $100 \mathrm{~mL}$. Hence, the investigators did demonstrate that the proportion of participants reaching the $100 \mathrm{~mL}$ improvement in the triple therapy group (42\%) was higher than in the beclometasone dipropionate(BDP)/ formoterol fumarate (FF) group (24\%), $(p<0.001)$ at week 26 and $38 \%$ versus $23 \%$ respectively at week 52 . The rate of pneumonia was around $3 \%$ in both treatment arms. The observed reduction in exacerbations is really driven by the difference in moderate exacerbations between the 2 groups as there were very few severe exacerbations. This group would not be considered a frequent exacerbation group as the average exacerbation rate for both treatment arms was 1.2 exacerbations and only $20 \%$ had 2 or more moderate or 1 severe exacerbation(s) in the prior 12 months. Based on the GOLD guidelines at the time of the design and recruitment for this study, July 2014, the majority of the patients were designated as group $D$, based on their low lung function. Most of these patients would now fall into group B (baseline average COPD Assessment test [CAT] score of 20). In fact, in this study, arguably it is the group that had 2 or more exacerbations in the previous 12 months, rate ratio (RR) 0.67 (95\% confidence interval [CI] 0.48-0.94; $p=0.019$ ) that drives the significant reduction in exacerbations compared to those with only 1 exacerbation in the prior 12 months, rate ratio 0.83 (95\% CI 0.67-1.02; $p=0.074)$. Interestingly, the average complete blood count eosinophil count in both groups was 250 eosinophils per microliter. There was no association between eosinophil levels and response to therapy noted in this study. Further, $75 \%$ of the participants in this study were on ICS/LABA combination therapy prior to entering the trial and during the 2-week run-in period, all participants were put on ICS/LABA therapy. Suissa has suggested that such designs introduce a selection bias based on run-in medication as one may sub-select a group who are good responders to ICS/ LABA drugs as they are the ones to make it through the run-in stage of the study up to randomization. ${ }^{20}$ Prior to entering the study, only $15 \%$ of the study participants were on LABA/LAMA combinations and $11 \%$ were on LAMA alone. It may be getting more difficult to recruit frequent exacerbators when the majority of patients are on maintenance therapies before entry into these trials. A recent analysis of the SPRIOMICS cohort indicated that the severe/frequent exacerbator group (2 or more moderate or 1 severe 
exacerbation [s]) is in fact a very small group ( 2\%) for patients who may already be on maintenance therapies such as ICSs/LABAs or LABAs/LAMAs. ${ }^{21}$

In the context of current GOLD guidelines, it is essential that studies compare single inhaler triple therapy formulations with LABA/LAMA combinations whether one is looking at improvements in symptoms and lung function or exacerbation rates. Fortunately, there are several studies (TRIBUTE, IMPACT, and ETHOS) currently being conducted that are designed to address these issues. ${ }^{22,23}$

\section{Abstract 2 \\ Single Inhaler Extrafine Triple Therapy Versus Long-Acting Muscarinic Antagonist Therapy for Chronic Obstructive Pulmonary Disease (TRINITY): A Double- Blind, Parallel Group, Randomised Controlled Trial}

Vestbo J, Papi A, Corradi M, et al. Lancet. 2017;389(10 082):1919-1929.

doi: https://doi.org/10.1016/S0140-6736(17)30188-5

\section{BACKGROUND:}

Limited data are available for the efficacy of triple therapy with two long-acting bronchodilators and an inhaled corticosteroid in chronic obstructive pulmonary disease (COPD). We compared treatment with extrafine beclometasone dipropionate, formoterol fumarate, and glycopyrronium bromide (BDP/FF/ GB; fixed triple) with tiotropium, and BDP/FF plus tiotropium (open triple).

\section{METHODS:}

For this double-blind, parallel-group, randomised, controlled trial, eligible patients had COPD, postbronchodilator forced expiratory volume in $1 \mathrm{~s}\left(\mathrm{FEV}_{1}\right)$ of less than $50 \%$, at least one moderate-to-severe COPD exacerbation in the previous 12 months, and a COPD Assessment Test total score of at least 10. After a 2-week run-in period receiving one inhalation per day via single-dose dry-powder inhaler of open-label $18 \mu \mathrm{g}$ tiotropium, patients were randomised (2:2:1) using a interactive response technology system to 52 weeks treatment with tiotropium, fixed triple, or open triple. Randomisation was stratified by country and severity of airflow limitation. The primary endpoint was moderate-to-severe COPD exacerbation rate. The key secondary endpoint was change from baseline in pre-dose $\mathrm{FEV}_{1}$ at week 52 . The trial is registered with ClinicalTrials.gov, number NCT01911364.

\section{FINDINGS:}

Between Jan 21, 2014, and March 18, 2016, 2691 patients received fixed triple $(n=1078)$, tiotropium ( $n=1075)$, or open triple ( $n=538)$. Moderate-to-severe exacerbation rates were 0.46 (95\% CI 0.41-0.51) for fixed triple, 0.57 (0.52-0.63) for tiotropium, and 0.45 (0.39-0.52) for open triple; fixed triple was superior to tiotropium (rate ratio 0.80 [95\% CI 0.69-0.92]; $p=0.0025$ ). For week 52 pre-dose $\mathrm{FEV}_{1}$, fixed triple was superior to tiotropium (mean difference $0.061 \mathrm{~L}$ [0.037 to 0.086]; $p<0.0001$ ) and non-inferior to open triple (-0.003L [-0.033 to 0.027]; $\mathrm{p}=0.85)$. Adverse events were reported by 594 (55\%) patients with fixed triple, 622 (58\%) with tiotropium, and 309 (58\%) with open triple.

\section{INTERPRETATION:}

In our TRINITY study, treatment with extrafine fixed triple therapy had clinical benefits compared with tiotropium in patients with symptomatic COPD, FEV 1 of less than $50 \%$, and a history of exacerbations.

FUNDING: Chiesi Farmaceutici SpA. PMID: 28385353

\section{Comments}

This study essentially has the same design as the TRILOGY trial except the comparators were tiotropium and an open triple combination that included BDP/ FF plus tiotropium. Tiotropium was used in the run-in period for all participants. Again, the majority of these patients would not be considered to be in Group D by current GOLD guidelines. The pre-study exacerbation rate was no more than 1.3 for any of the 3 treatment arms and again less than $20 \%$ of participants had had 2 or more exacerbations in the previous 12 months. Blood eosinophil concentration was about 200 per microliter across all groups. Eosinophil counts did not appear to be associated with treatment effects with regard to lung function. The authors reported that both triple therapies demonstrated greatest reductions in exacerbations for those with eosinophil counts of at least $2 \%, \mathrm{RR} 0.70$ 
(95\% CI 0.58-0.85) for fixed and 0.69 (0.55-0.87) for open triple compared to less than $2 \%, 0.93$ (0.751.17 ) and 0.91(0.69-1.20) respectively. Using absolute eosinophil counts of greater than 200 , the RR was 0.64 (0.51-0.81) and 0.62 (0.47-0.83) respectively. The rate of pneumonia was low at $2 \%$ for both triple therapy arms and $1 \%$ for the tiotropium arm. As with the TRILOGY study, the most relevant comparator would be a LAMA/ LABA combination. Between this issue and the fact that this is not truly a frequent exacerbator group it is not possible to extend these findings to the frequent exacerbator group. Certainly, the sub-analysis is helpful but the numbers of frequent exacerbators was very small. One question is whether these trials suggest that we need to revisit whether forced expiratory volume in 1 second $\left(\mathrm{FEV}_{1}\right)$ should be reincorporated into the GOLD guidelines treatment decision paradigm to decide if patients with a low $\mathrm{FEV}_{1}$, regardless of exacerbation frequency, should be immediately started on triple therapy rather than LABA/LAMA. As mentioned above, we will gain some insights into this question from results from current studies that are underway that are comparing single inhaler triple therapy with various single inhaler dual bronchodilators. ${ }^{23}$

\section{Abstract 3 \\ FULFIL Trial: Once-Daily Triple Therapy for Patients with Chronic Obstructive Pulmonary Disease}

Lipson DA, Barnacle H, Birk R, et al. Am J Respir Crit Care Med. 2017;196 (4):438-446.

doi: https://doi.org/10.1164/rccm.201703-04490C

\section{RATIONALE:}

Randomized data comparing triple therapy with dual inhaled corticosteroid (ICS)/long-acting $\beta 2$-agonist (LABA) therapy in patients with chronic obstructive pulmonary disease (COPD) are limited.

\section{OBJECTIVES:}

We compared the effects of once-daily triple therapy on lung function and health-related quality of life with twice-daily ICS/LABA therapy in patients with COPD.

\section{METHODS:}

The FULFIL (Lung Function and Quality of Life Assessment in Chronic Obstructive Pulmonary
Disease with Closed Triple Therapy) trial was a randomized, double-blind, double-dummy study comparing 24 weeks of once-daily triple therapy (fluticasone furoate/umeclidinium/vilanterol 100 $\mathrm{\mu g} /$ $62.5 \mu \mathrm{g} / 25 \mu \mathrm{g}$; ELLIPTA inhaler) with twice-daily ICS/ LABA therapy (budesonide/formoterol 400 $\mu \mathrm{g} / 12 \mu \mathrm{g}$; Turbuhaler). A patient subgroup remained on blinded treatment for up to 52 weeks. Co-primary endpoints were change from baseline in trough $\mathrm{FEV}_{1}$ and in St. George's Respiratory Questionnaire (SGRQ) total score at Week 24.

\section{MEASUREMENTS AND MAIN RESULTS:}

In the intent-to-treat population $(\mathrm{n}=1,810)$ at Week 24 for triple therapy $(n=911)$ and ICS/LABA therapy $(n=899)$, mean changes from baseline in $\mathrm{FEV}_{1}$ were $142 \mathrm{ml}$ (95\% confidence interval [CI], 126 to 158) and $-29 \mathrm{ml}$ ( $95 \% \mathrm{CI},-46$ to -13 ), respectively, and mean changes from baseline in SGRQ scores were -6.6 units (95\% CI, -7.4 to -5.7$)$ and -4.3 units (95\% CI, -5.2 to -3.4), respectively. For both endpoints, the betweengroup differences were statistically significant $(P<0.001)$. There was a statistically significant reduction in moderate/severe exacerbation rate with triple therapy versus dual ICS/LABA therapy (35\% reduction; $95 \% \mathrm{CI}, 14-51 ; P=0.002)$. The safety profile of triple therapy reflected the known profiles of the components.

\section{CONCLUSIONS:}

These results support the benefits of single-inhaler triple therapy compared with ICS/LABA therapy in patients with advanced COPD.

\section{KEYWORDS:}

chronic obstructive pulmonary disease; health-related quality of life; lung function; single-inhaler triple therapy

\section{Comments}

This trial is interesting in that the investigators opted to compare this once per day single inhaler triple combination with an ICS/LABA combination that was different from the mono-components of the triple and had twice-a-day dosing. It is interesting that the once-aday formulation seemed to be better than the twice-a-day formulation. Details are not provided but presumably the trough $\mathrm{FEV}_{1}$ was measured after 24 hours and 2 doses of 
the budesonide (BUD) /formoterol (FOR) formulation. This was a study largely conducted in Europe where budesonide is indeed one of the most commonly used ICS/LABA combination formulations used. In contrast to the 2 previous studies, that put all patients on the same medication during the run-in period, this study allowed patients to remain on their pre-study maintenance therapy up to the time of randomization. The intent was to make the study as "real world" as possible. This study was started January 2015 and utilized the older GOLD classification schemes. The mean $\mathrm{FEV}_{1}$ was around $1.4 \mathrm{~L}$ (46\% of predicted). Only $38 \%$ of this cohort had 2 or more exacerbations and $34 \%$ had no history of exacerbations in the previous 12 months. Applying the current GOLD classification scheme, $62 \%$ of the patients in this study would be considered Group B. Further, the extended cohort of the study only had 430 patients out of the 1810 patients who were part of the initial 24-week study. In the first 24 weeks of the study there were 19 reports of pneumonia in the triple therapy group ( $\mathrm{n}=911)(2.2 \%)$ versus 7 in the BUD/FOR group $(\mathrm{n}=899)(0.8 \%)$ and in the extension cohort there were $4 / 210$ and $4 / 220$ respectively. It is not clear if patients who experienced pneumonia were discontinued in the study. As in the 2 studies above, the question remains as to whether LAMA/LABA combinations might demonstrate similar benefits compared to the single inhaler triple combination, not only in terms of lung function and quality of life measures, but also in terms of exacerbation reduction in Group B and/or Group D patients.

\section{Abstract 4 \\ Mepolizumab for Eosinophilic Chronic Obstructive Pulmonary Disease}

Pavord ID, Chanez P, Criner GJ, et al. N Engl J Med.

2017;377(17):1613-1629.

doi: https://doi.org/10.1056/NEJMoa1708208

\section{BACKGROUND:}

Patients with chronic obstructive pulmonary disease (COPD) with an eosinophilic phenotype may benefit from treatment with mepolizumab, a monoclonal antibody directed against interleukin-5.

\section{METHODS:}

We performed two Phase 3, randomized, placebo- controlled, double-blind, parallel-group trials comparing mepolizumab (100 $\mathrm{mg}$ in METREX, 100 or $300 \mathrm{mg}$ in METREO) with placebo, given as a subcutaneous injection every 4 weeks for 52 weeks in patients with COPD who had a history of moderate or severe exacerbations while taking inhaled glucocorticoid-based triple maintenance therapy. In METREX, unselected patients in the modified intention-to-treat population with an eosinophilic phenotype were stratified according to blood eosinophil count $(\geq 150$ per cubic millimeter at screening or $\geq 300$ per cubic millimeter during the previous year). In METREO, all patients had a blood eosinophil count of at least 150 per cubic millimeter at screening or at least 300 per cubic millimeter during the previous year. The primary end point was the annual rate of moderate or severe exacerbations. Safety was also assessed.

\section{RESULTS:}

In METREX, the mean annual rate of moderate or severe exacerbations in the modified intention-totreat population with an eosinophilic phenotype (462 patients) was 1.40 per year in the mepolizumab group versus 1.71 per year in the placebo group (rate ratio, 0.82; 95\% confidence interval [CI], 0.68 to 0.98 ; adjusted $P=0.04$ ); no significant betweengroup differences were found in the overall modified intention-to-treat population (836 patients) (rate ratio, 0.98 ; $95 \% \mathrm{CI}, 0.85$ to 1.12 ; adjusted $P>0.99$ ). In METREO, the mean annual rate of moderate or severe exacerbations was 1.19 per year in the 100mg mepolizumab group, 1.27 per year in the $300-\mathrm{mg}$ mepolizumab group, and 1.49 per year in the placebo group. The rate ratios for exacerbations in the $100-\mathrm{mg}$ and 300-mg mepolizumab groups versus the placebo group were 0.80 (95\% CI, 0.65 to 0.98 ; adjusted $P=0.07$ ) and 0.86 (95\% CI, 0.70 to 1.05 ; adjusted $P=0.14$ ), respectively. A greater effect of mepolizumab, as compared with placebo, on the annual rate of moderate or severe exacerbations was found among patients with higher blood eosinophil counts at screening. The safety profile of mepolizumab was similar to that of placebo.

\section{CONCLUSIONS:}

Mepolizumab at a dose of $100 \mathrm{mg}$ was associated with a lower annual rate of moderate or severe exacerbations than placebo among patients with COPD and an 
eosinophilic phenotype. This finding suggests that eosinophilic airway inflammation contributes to COPD exacerbations. (Funded by GlaxoSmithKline; METREX and METREO

\section{Comments}

This study is based on the premise that there is at least a subset of COPD patients whose exacerbations are associated with eosinophilic inflammation. Rather than trying to use a constellation of clinical characteristics and biomarkers to define a so-called asthma/COPD overlap phenotype, the focus on treatable traits (high eosinophil count) may simplify decision-making. Investigators from this study and others have suggested that ICSs appear to have the greatest benefit in COPD patients with peripheral blood absolute eosinophil counts above 300 and at least 2 exacerbations in the previous 12 months. ${ }^{3-5,19}$ In both trials, at least $94 \%$ of patients fell into current Group D classification with 2 moderate or 1 severe exacerbation(s). It is not clear from the study design whether patients were all converted over to the same ICS/LABA/LAMA combination of drugs. In the METREX study the unselected population included those who met the eosinophil criteria and a group that had eosinophil counts less than $150 / \mu \mathrm{L}$. Adding the non-eosinophilic group in the analysis rendered the results in the METREX trial statistically insignificant. Interestingly, the placebo group had slightly higher baseline eosinophil levels compared to the mepolizumab group, (290 versus $260 / \mu \mathrm{L}$ ) for those with an eosinophil count of greater than $150 / \mu \mathrm{L}$ at time of study entry and 360 versus $200 / \mu \mathrm{L}$ respectively for those who had peripheral blood eosinophil counts greater than 300 in the previous 12 months. It is worth noting that the exacerbation rates in the placebo groups during 12 months before the study (2.5 to 2.6) were significantly higher than during the 12 months of the trial (around 1.5-1.7). The authors propose that this might reflect the study effect, that patients were using the medications more regularly and appropriately during the trial. In the METREO study they did not meet statistical significance for the primary endpoint for either dose. This indeed emphasizes the need to ensure that patients are compliant and adherent with standard therapies before making decisions about trials with biologics. The $100 \mathrm{mg}$ dose appeared to be at least as, if not more, effective than the $300 \mathrm{mg}$ dose in the METREO study. The authors also noted higher rates of pneumonia in these studies (9\%-11\%) compared to other reports. They offered that this could be related to the relatively high dose ICS use in this refractory group of COPD patients with frequent exacerbations. Further, there were so few exacerbation events that they were unable to assess reductions of severe exacerbations or mortality in this study. None of the secondary endpoints studied, including St George's Respiratory Questionnaire, CAT score, time to first moderate to severe exacerbation, and exacerbations leading to emergency department visits or hospitalizations reached statistical significance. The bottom line is that it is likely that there is a subset of COPD patients who may or may not meet the criteria for asthma/COPD overlap but appear to have treatable traits including frequent exacerbations of 2 or more moderate exacerbations or 1 hospitalization in the previous 12 months and an eosinophil count of 150 per microliter at time of evaluation regardless of their steroid use or at least a 300 eosinophil per microliter historically that should be considered for treatment with mepolizumab after having failed high dose inhaled corticosteroids and or systemic corticosteroids. 


\section{References}

1. Singh D. Pharmacological treatment for COPD; GOLD 2017 changes direction. Br J Clin Pharmacol. 2017;83(5):935-937. doi: https://doi.org/10.1111/bcp.13212

2. Kolsum U, Ravi A, Hitchen P, Maddi S, Southworth T, Singh D. Clinical characteristics of eosinophilic COPD versus COPD patients with a history of asthma. Respir Res. 2017;18(1):73. doi: https://doi.org/10.1186/s12931-017-0559-0

3. Pavord ID, Lettis S, Locantore N, et al. Blood eosinophils and inhaled corticosteroid/long-acting beta-2 agonist efficacy in COPD. Thorax. 2016.71(2):118-125.

doi: https://doi.org/10.1136/thoraxjnl-2015-207021

4. Barnes NC, SharmaR, Lettis S, Calverley PMA. Blood eosinophils as a marker of response to inhaled corticosteroids in COPD. Eur Respir J. 2016;47(5):1374-1382.

doi: https://doi.org/10.1183/13993003.01370-2015

5. Bafadhel M,Greening NJ, Harvey-Dustan TC, et al. Blood eosinophils and outcomes in severe hospitalized exacerbations of COPD. Chest. 2016; 150(2):320-328.

doi: https://doi.org/10.1016/j.chest.2016.01.026

6. Calverley PM, Tetzlaff K, Vogelmeier C, et al. Eosinophilia, frequent exacerbations, and steroid response in chronic obstructive pulmonary disease. Am J Respir Crit Care Med. 2017;196(9): 1219-1221.

doi: https://doi.org/10.1164/rccm.201612-2525LE

7. Crim C, Calverley PMA, Anderson JA, et al. Pneumonia risk with inhaled fluticasone furoate and vilanterol in COPD patients with moderate airflow limitation: The SUMMIT trial. Respir Med. 2017;131:27-34. doi: https://doi.org/10.1016/j.rmed.2017.07.060

8. Suissa S, Coulombe J, Ernst P. Discontinuation of inhaled corticosteroids in COPD and the risk reduction of pneumonia. Chest. 2015;148(5):1177-1183.

doi: https://doi.org/10.1378/chest.15-0627

9. Crim C, Dransfield MT, Boirbeau J, et al. Pneumonia risk with inhaled fluticasone furoate and vilanterol compared with vilanterol alone in patients with COPD. Ann Am Thorac Soc. 2015; 12(1):27-34.

doi: https://doi.org/10.1513/AnnalsATS.201409-413OC

10. Stanbrook MB. ACP Journal Club. Review: In COPD, fluticasone or budesonide increases serious pneumonia but not mortality. Ann Intern Med. 2014; 161(4): JC8.

doi: https://doi.org/10.7326/0003-4819-161-4-201408190-02008

11. Suissa S, Patenaude V, Lapi F, Ernst P. Inhaled corticosteroids in COPD and the risk of serious pneumonia. Thorax. 2013; 68(11):1029-1036.

doi: https://doi.org/10.1136/thoraxjnl-2012-202872
12. Wedzicha JA, Banerji D, Vogelmeier CF. Indacaterolglycopyrronium for COPD. N Engl J Med. 2016; 375(9): 899-900.

13. Wedzicha JA, Bannerji D, Chapman KR, et al. Indacaterolglycopyrronium versus salmeterol-fluticasone for COPD. N Engl J Med. 2016; 374(23):2222-2234.

doi: https://doi.org/10.1056/NEJMoa 1516385

14. Wedzicha JA, Agusti A, Donaldson C, Chuecos F, Lamarca R, Garcia Gil E. Effect of aclidinium bromide on exacerbations in patients with moderate-to-severe COPD: A pooled analysis of five phase III, randomized, placebo-controlled studies. COPD. 2016; 13(6): 669-676.

doi: https://doi.org/10.3109/15412555.2016.1170111

15. Singh D, Maleki-Yazdi MR, Tombs L, Iqbal A, Fahy WA, Naya I. Prevention of clinically important deteriorations in COPD with umeclidinium/vilanterol. Int J Chron Obstruct Pulmon Dis. 2016;11:1413-1424. doi: https://doi.org/10.2147/COPD.S101612

16. Singh D, Schmidt O, Bjermer L, Gronke L, Voss F, Ferguson GT. Effects of tiotropium + olodaterol versus tiotropium or placebo by COPD disease severity and previous treatment history in the OTEMTO(R) studies. Respir Res. 2016; 17(1): 73. doi: https://doi.org/10.1186/s12931-016-0387-7

17. Wedzicha JA, DeCramer M, Ficker JM, et al. Analysis of chronic obstructive pulmonary disease exacerbations with the dual bronchodilator QVA149 compared with glycopyrronium and tiotropium (SPARK): a randomised, double-blind, parallel-group study. Lancet Respir Med. 2013;1(3):199-209. doi: https://doi.org/10.1016/S2213-2600(13)70052-3

18. Brightling CE, Bleecker ER, Panettieri RA Jr, et al. Benralizumab for chronic obstructive pulmonary disease and sputum eosinophilia: a randomised, double-blind, placebo-controlled, phase 2a study. Lancet Respir Med. 2014; 2(11):891-901. doi: https://doi.org/10.1016/S2213-2600(14)70187-0

19. Calverley PMA. A light in the darkness? The FLAME Trial, blood eosinophils, and chronic obstructive pulmonary disease. Am J Respir Crit Care Med. 2017;195(9): 1125-1127. doi: https://doi.org/10.1164/rccm.201703-0560ED

20. Suissa S. Run-in bias in randomised trials: The case of COPD medications. Eur Respir J. 2017; 49(6).

doi: https://doi.org/10.1183/13993003.00361-2017

21. Han MK, Quibrera PM, Carretta EE, et al. Frequency of exacerbations in patients with chronic obstructive pulmonary disease: an analysis of the SPIROMICS cohort. Lancet Respir Med. 2017; 5(8):619-626.

doi: https://doi.org/10.1016/S2213-2600(17)30207-2

$\overline{\text { 22. Pascoe SJ, Lipson DA, Locantore N, et al. A phase III randomised }}$ controlled trial of single-dose triple therapy in COPD: the IMPACT protocol. Eur Respir J. 2016;48(2):320-30. doi: https://doi.org/10.1183/13993003.02165-2015 
23. Calverley PMA, Magnussen H, Miravitlles M, Wedzicha JA. Triple therapy in COPD: What we know and what we don't. COPD. 2017;14(6):648-662.

doi: https://doi.org/10.1080/15412555.2017.1389875 